\title{
X-rays and dynamics
}

\section{Silvia Pellegrini} Department of Astronomy, University of Bologna, via Ranzani 1, 40127 Bologna, Italy
email: silvia.pellegrini@unibo.it

\begin{abstract}
The hot X-ray emitting interstellar medium of early type galaxies can be used in principle as a total mass tracer and a tool to determine the stellar orbital anisotropy, based on the hypothesis of hydrostatic equilibrium for it. Here the effects that deviations from equilibrium have on both estimates are shown, and a comparison is made with cases for which accurate optical and X-ray information are available.
\end{abstract}

Keywords. galaxies: elliptical and lenticular, $\mathrm{cD}$ - galaxies: fundamental parameters - galaxies: halos - galaxies: ISM - galaxies: kinematics and dynamics - galaxies: structure

The mass distribution and amount and the internal kinematics of an early type galaxy can be derived from X-ray observations of its hot gas properties (density and temperature profiles) and optical observations of the motions of stellar objects (stars, planetary nebulae and globular clusters; e.g., Ciotti \& Pellegrini 2004). Recently, a lot of progress in data quality and analysis methods has allowed to exploit X-ray and optical information extending over similar radial ranges, and out to 10-15 effective radii (e.g., Humphrey et al. 2006, Coccato et al. 2009). One can then investigate whether there is agreement or contradiction between the results from the X-ray and optical analysis used independently, to establish the robustness of each method taken separately. For example, the mass-anisotropy degeneracy may affect optical estimates, while hydrostatic equilibrium (a necessary condition to exploit the X-ray information) may not hold due to bulk flows, or may require pressure terms other than thermal and unaccounted for. A general result concerning possible deviations of X-ray estimates from true values, that is independent of assumptions about the underlying mass profile or the internal orbital distribution, is the following: the deviations are in the sense of mass underestimates accompanied by orbital anisotropy overestimates, and viceversa. From hydrodynamical models of the hot gas flow evolution for representative galaxies one derives mass underestimates for infalling gas and overestimates for outflows (Pellegrini \& Ciotti 2006). This could explain some observed discrepancies between $\mathrm{X}$-ray and optical estimates of dynamical quantities, as have been noted for example within one effective radius for galaxies with detailed optical and X-ray information (e.g., NGC4472, NGC4649 and NGC1407; Ciotti \& Pellegrini 2004, Hwang et al. 2008, Romanowsky et al. 2009).

\section{References}

Ciotti, L. \& Pellegrini, S. 2004, MNRAS 350, 609

Coccato, L. et al. 2009, MNRAS 394, 1249

Humphrey, P. J. et al. 2006, ApJ 646, 899

Hwang, H. S. et al. 2008, ApJ 674, 869

Pellegrini, S. \& Ciotti, L. 2006, MNRAS 370, 1797

Romanowsky, A. et al. 2009, AJ 137, 4956 